\title{
A protocol for the emergency department management of acute undifferentiated febrile illness in India
}

Sudhagar Thangarasu', Piruthiviraj Natarajan², Parivalavan Rajavelu², Arjun Rajagopalan ${ }^{3}$ and Jeremy S Seelinger Devey ${ }^{4^{*}}$

\begin{abstract}
Background: Fever is a common presenting complaint in the developing world, but there is a paucity of literature to guide investigation and treatment of the adult patient presenting with fever and no localizing symptoms.

Objective: The objective of this study was to devise a standardized protocol for the evaluation and treatment of febrile adult patients who have no localizing symptoms in order to reduce unnecessary testing and inappropriate antimicrobial use. After devising the protocol, a pilot study was performed to assess its feasibility in the emergency department.

Methods: A protocol was formulated for adult patients presenting with fever who had no clinical evidence of sepsis and no localizing symptoms to suggest the etiology of their fever. Investigations were based on duration of fever with no investigations indicated prior to day 3. Treatment was guided by results of investigations. A pilot study was performed after protocol implementation, wherein data were collected on successive adult patients presenting with fever.

Results: During the 6-week study period, 342 patients presented with fever, 209 of whom fit the parameters of the protocol, with 113 of these patients presenting on the 1st or 2 nd day of fever. All patients experienced defervescence of fever, with ten patients being lost to follow-up. Of the patients presenting on day 1 or 2 of fever, 75.2\% (85/113) defervesced without the need for testing; 53.1\% (60/113) experienced defervescence without the need for antimicrobial therapy.

Conclusion: Implementation of this rational, standardized protocol for the assessment and treatment of stable adult patients presenting with acute undifferentiated febrile illness can lead to reduced rates of testing and antimicrobial use. A prospective, controlled trial will be required to confirm these findings and to assess additional safety outcome measures.
\end{abstract}

\section{Introduction}

Fever is a common presenting complaint in the developing world and is the most common presentation to the Emergency Department (ED) at our institution, Sundaram Medical Foundation (SMF) in Chennai, India [1]. Febrile illness can be localized to organ systems or nonlocalized, commonly referred to as acute undifferentiated febrile illness (AUFI). In the Western world, AUFI

\footnotetext{
* Correspondence: jsdevey@gmail.com

${ }^{4}$ Dept. of International Emergency Medicine, Long Island Jewish Medical

Center, 270-05 $76^{\text {th }}$ Ave., New Hyde Park, NY 11040, USA

Full list of author information is available at the end of the article
}

is often due to self-limited viral conditions. However, in the developing world, the differential diagnosis for AUFI includes potentially significant illnesses such as malaria, dengue fever, enteric fever, leptospirosis, rickettsiosis, hantavirus, and Japanese encephalitis [2-10]. There is a paucity of literature on the appropriate evaluation of adult fever patients without localizing symptoms in the ED [11]. In the absence of established protocols, patients may be subjected to unnecessary investigations at considerable cost and the inappropriate prescribing of antimicrobial therapy $[12,13]$. In the following, we describe a protocol that was formulated and implemented in the 
SMF ED to evaluate adult patients presenting with nonlocalizing fever.

\section{Objective}

The aim of this pilot study was to devise and implement a protocol for the management of stable adult patients presenting to the emergency department with fever as their chief complaint and no localizing symptoms. The overarching goal of the protocol was to standardize the approach to such patients in a way that reduced unnecessary testing and inappropriate use of antibiotics. Additional goals, such as improving time to fever resolution, reduction in hospital admission rate, and reduction in mortality, while also ultimately desirable, were not assessed in this study.

\section{Methods}

A protocol for the management of stable adult patients presenting to the SMF ED with a chief complaint of fever was devised according to the local infectious epidemiology by SMF emergency physicians in consultation with SMF medicine consultants and is presented in Figure 1. All adult patients aged 17 and older with a presenting complaint of fever but without localizing symptoms were considered for evaluation by the protocol. Patients with localizing symptoms that suggested the etiology of fever and those meeting criteria for severe sepsis or septic shock were excluded. Eligible

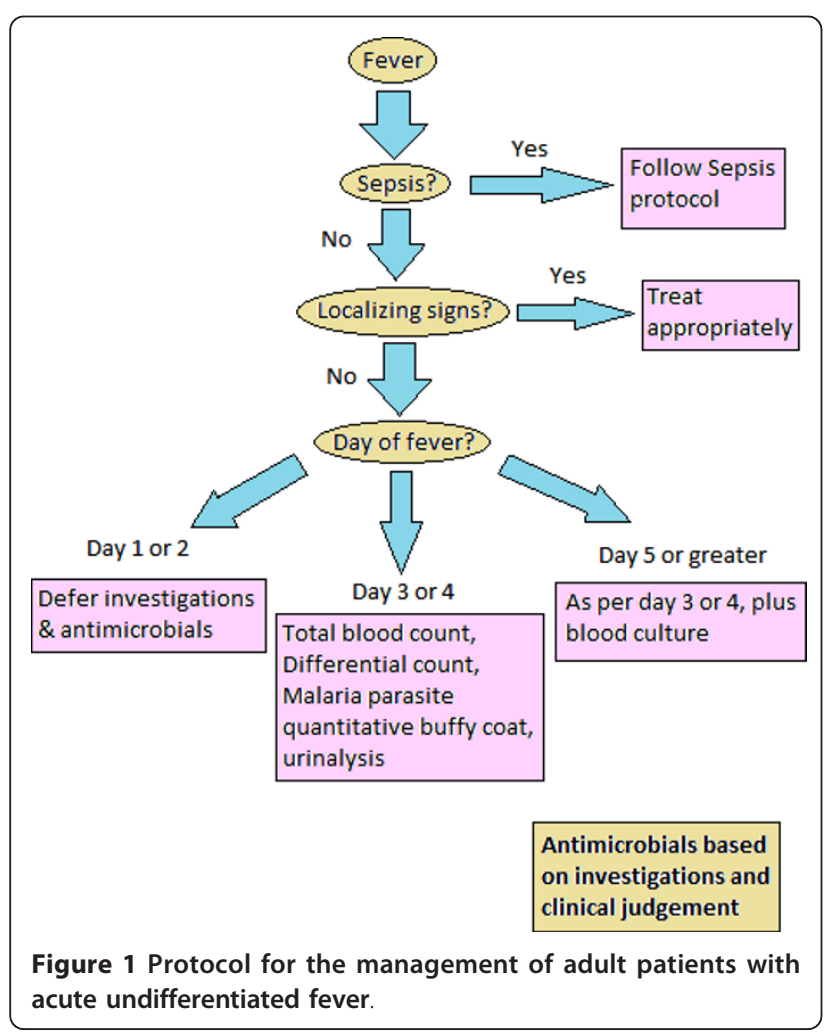

patients were managed either by the protocol or as deemed most appropriate by the evaluating physician. Under the protocol, if an eligible patient was stable and had had less than 3 days of fever, all investigations and antimicrobial therapy were deferred, and the patient was prescribed antipyretics and asked to return to the ED on the 3rd day of fever if it persisted. Patients presenting on days 3 or 4 of fever had total blood count, differential count, malaria parasite quantitative buffy coat test, and urinalysis performed. Patients presenting on day 5 or greater of fever additionally had a blood culture performed. All patients were then treated according to the results of investigations as deemed appropriate.

In order to assess the feasibility of the implementation of this protocol, data were prospectively collected on all eligible patients presenting to the SMF ED between 1 August 2008 and 15 September 2008. Data collected included day of fever at presentation, day of fever resolution, investigations performed, antimicrobial therapy received or not, and final diagnosis. Thirty-day followup was performed by phone interview and examination of medical records to assess final outcomes. The study protocol was reviewed and approved by the IRB at Sundaram Medical Foundation.

\section{Results}

During the study period 342 patients presented with fever. Of these, $6(1.8 \%)$ met the clinical definition of sepsis and were treated according to sepsis protocol, and 127 (37.1\%) had localizing symptoms to suggest an etiology for their fever. This left 209 patients (61.1\%) with AUFI eligible for the protocol. The majority of these patients were presenting on the 1st or 2nd day of fever (Figure 2).

Of the 113 AUFI patients who presented within the first 2 days of fever, $57.5 \%$ (65/113) were treated according to the protocol and received no investigations (Table 1). Of these, 75.4\% (49/65) experienced spontaneous defervescence, while the remainder underwent investigation per the protocol at the 3- and 5-day follow-up. Among the 48 patients presenting within the first 2 days of fever who underwent investigations outside of the protocol, all experienced defervescence. The investigations were contributory to patient management in $25.0 \%(12 / 48)$ of these cases and did not change management in the remaining $75.0 \%$ (36/48). Four patients were lost to follow-up. Investigations were ultimately unnecessary in $75.2 \%$ of patients ( 49 who defervesced without investigation plus 36 who had non-contributory investigations and defervesced out of 113 patients) presenting on the 1st or 2nd day of fever.

Antimicrobial therapy was prescribed to 35 of the 113 AUFI patients who initially presented within the first 2 


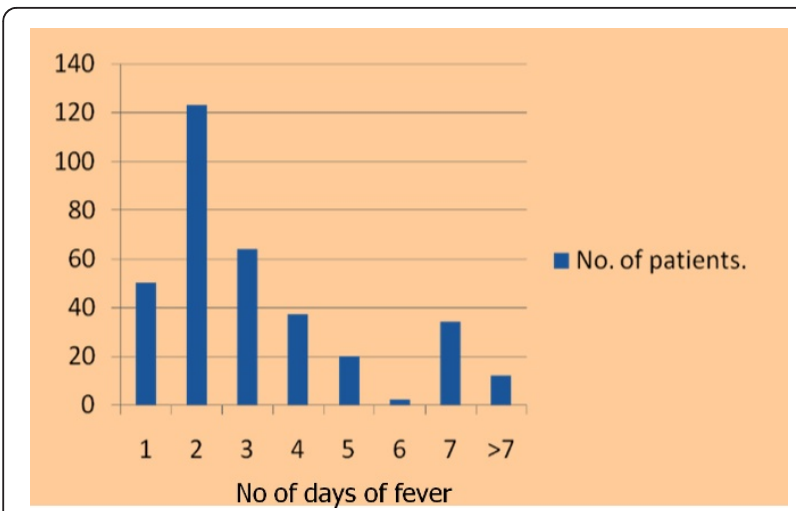

Figure 2 Day of fever at the time of presentation.

days of fever and ultimately received at a later date by 15 additional patients. Three patients were lost to follow-up. Of the patients, 53.1\% (60/113) experienced defervescence without the need for antimicrobial therapy.

All patients experienced resolution of fever, with ten being lost to follow-up. The final etiology of fever was never determined in the majority of cases (Figure 3 ).

\section{Discussion}

Given the relative frequency with which emergency physicians in India encounter patients with acute undifferentiated febrile illness, it is in our interest to develop a

\section{Table 1 Outcomes of stable adult patients with acute undifferentiated febrile illness presenting on day 1 or 2 of fever}

\begin{tabular}{lll}
\hline & Number & Percent* \\
\hline Eligible patients, day 1 or 2 of fever & 113 & $100 \%$ \\
Received investigations initially & 48 & $42.5 \%$ \\
$\quad$ Investigations contributory & 12 & $25 \%$ \\
$\quad$ Investigations non-contributory & 36 & $75 \%$ \\
Did not receive investigations initially & 65 & $57.5 \%$ \\
$\quad$ Defervesced without need for & 49 & $75.4 \%$ \\
$\quad$ investigations & & \\
$\quad$ Eventually investigated as per protocol & 12 & $12.7 \%$ \\
$\quad$ Lost to follow-up & 4 & $6.2 \%$ \\
Total defervesced without need for & $\mathbf{8 5}$ & $\mathbf{7 5 . 2} \%$ \\
investigations & & \\
Received antimicrobials initially & 35 & $31 \%$ \\
Did not receive antimicrobials initially & 78 & $69 \%$ \\
$\quad$ Defervesced without need for & 60 & $87 \%$ \\
$\quad$ antimicrobials & & \\
$\quad$ Eventually required antimicrobials & 15 & $19.2 \%$ \\
$\quad$ Lost to follow-up & 3 & $3.8 \%$ \\
Total defervesced without need for & $\mathbf{6 0}$ & $\mathbf{5 3 . 1 \%}$ \\
antimicrobials
\end{tabular}

*Percentages calculated using subcategory as denominator.

Bold items highlighted to illustrate the potential for reduction in unnecessary investigations and inappropriate antimicrobial therapy.

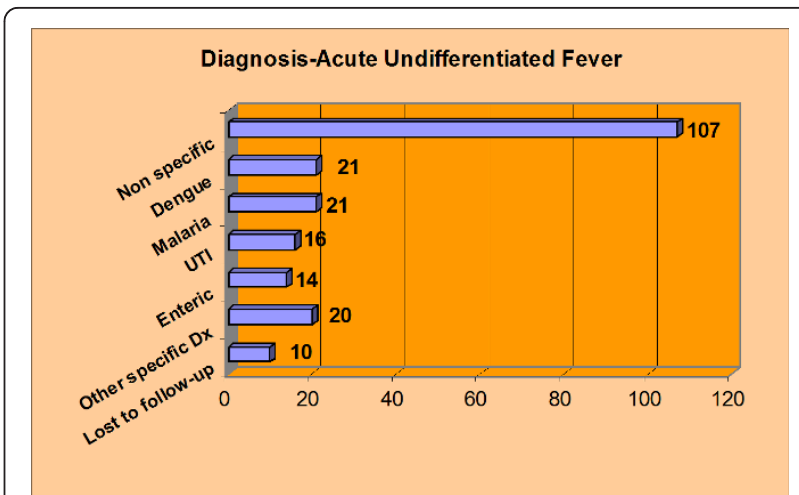

Figure 3 Final diagnosis of adult patients with acute undifferentiated fever.

standardized approach to evaluating these patients. Evidence-based protocols have been shown to be costeffective [14] and improve mortality [15] in the emergency department setting. This protocol has the more modest goals of reducing costs, avoiding unnecessary testing and inappropriate therapies, and reducing antibiotic resistance and rates of misdiagnosis. We have described a protocol that represents a rational, graded approach to stable adult patients with AUFI that is informed by local infectious epidemiology [2]. In this pilot study, investigations were or could have been avoided in $75.2 \%$ of patients, and antimicrobial therapy was unnecessary for fever resolution in $53.1 \%$ of eligible patients with fever of $<3$ days duration. These data suggest that this protocol has the potential to reduce unnecessary testing and inappropriate antimicrobial use. A prospective trial will need to be carried out both to corroborate these findings as well as to investigate the ability of the protocol to influence additional outcome measures such as time to fever resolution, hospital admission rate, and mortality rate.

\section{Conclusion}

Implementation of a rational, standardized protocol for the assessment of stable adult patients with acute undifferentiated febrile illness in this south Indian emergency department demonstrates a potential to lower rates of unnecessary testing and antimicrobial use. The protocol will need to be prospectively validated in a controlled fashion in order to confirm these findings as well as to assess its safety.

\section{Author's information}

TS is a Resident Physician in Internal Medicine, University of Pittsburgh Medical Center-Mercy Hospital. NP is Senior House Officer in Emergency Medicine at Sundaram Medical Foundation. PVR is Head of Department, Department of Emergency Medicine at Sundaram 
Medical Foundation. AR is Medical Director and Head of Department, Department of Surgery at Sundaram Medical Foundation. JSD is International Emergency Medicine Fellow at Long Island Jewish Medical Center.

\section{List of abbreviations}

ED: Emergency department; SMF: Sundaram Medical Foundation, Chennai, Tamil Nadu, India; AUFI: acute undifferentiated febrile illness; IRB: institutional review board.

\section{Acknowledgements}

We thank Dr. D.V. Nagendra Naidu, who helped with the initial design of the study; we thank Drs. T. Girija, V. Seshadri, and M. Swamikannu, who were involved in the protocol design.

\section{Author details}

Dept. of Internal Medicine, University of Pittsburgh Medical Center-Mercy Hospital, 1400 Locust Street, Pittsburgh, PA 15206, USA ²Dept. of Emergency Medicine, Sundaram Medical Foundation, Shanthi Colony, $4^{\text {th }}$ Avenue, Anna Nagar, Chennai - 600040, India ${ }^{3}$ Dept. of Surgery, Sundaram Medical Foundation, Shanthi Colony, $4^{\text {th }}$ Avenue, Anna Nagar, Chennai - 600040, India ${ }^{4}$ Dept. of International Emergency Medicine, Long Island Jewish Medical Center, 270-05 $76^{\text {th }}$ Ave., New Hyde Park, NY 11040, USA

\section{Authors' contributions}

TS designed the study and collected data; NP collected data and followed up patients, PVR designed the study, supervised data collection and edited manuscript; and AR supervised the study design and edited the manuscript. JSD reviewed the available literature, edited for content, and prepared the manuscript. All authors read and approved the final manuscript.

\section{Competing interests}

The authors declare that they have no competing interests.

Received: 5 May 2011 Accepted: 5 September 2011

Published: 5 September 2011

\section{References}

1. Anthony DR, Balsari S, Clark S, Straff DJ, Rajavelu P, Rajagopalan A: The EMcounter Project: A Study of the Epidemiology of Medical Emergencies in India [abstract]. Ann Emerg Med 2007, 50(3):S129-S130.

2. Chrispal A, Boorugu H, Gopinath KG, Chandy S, Prakash JA, Thomas EM, Abraham AM, Abraham OC, Thomas K: Acute undifferentiated febrile illness in adult hospitalized patients: the disease spectrum and diagnostic predictors - an experience from a tertiary care hospital in South India. Trop Doct 2010, 40(4):230-4.

3. Manock SR, Jacobsen KH, de Bravo NB, Russell KL, Negrete M, Olson JG, Sanchez JL, Blair PJ, Smalligan RD, Quist BK, Espín JF, Espinoza WR, MacCormick F, Fleming LC, Kochel T: Etiology of acute undifferentiated febrile illness in the Amazon basin of Ecuador. Am J Trop Med Hyg 2009, 81(1):146-51.

4. Gasem MH, Wagenaar JF, Goris MG, Adi MS, Isbandrio BB, Hartskeerl RA, Rolain JM, Raoult D, van Gorp EC: Murine typhus and leptospirosis as causes of acute undifferentiated fever, Indonesia. Emerg Infect Dis 2009, 15(6):975-7.

5. Chandy S, Yoshimatsu K, Boorugu HK, Chrispal A, Thomas K, Peedicayil A, Abraham P, Arikawa J, Sridharan G: Acute febrile illness caused by hantavirus: serological and molecular evidence from India. Trans $R$ Soc Trop Med Hyg 2009, 103(4):407-12.

6. Kumar R, Tripathi P, Tripathi S, Kanodia A, Pant S, Venkatesh V: Prevalence and clinical differentiation of dengue fever in children in northern India. Infection 2008, 36(5):444-9.

7. Phuong HL, de Vries PJ, Nga TT, Giao PT, Hung le Q, Binh TQ, Nam NV, Nagelkerke N, Kager PA: Dengue as a cause of acute undifferentiated fever in Vietnam. BMC Infect Dis 2006, 6:123.

8. Suttinont C, Losuwanaluk K, Niwatayakul K, Hoontrakul S, Intaranongpai W, Silpasakorn S, Suwancharoen D, Panlar P, Saisongkorh W, Rolain JM, Raoult D, Suputtamongkol Y: Causes of acute, undifferentiated, febrile illness in rural Thailand: results of a prospective observational study. Ann Trop Med Parasitol 2006, 100(4):363-70.

9. Leelarasamee A, Chupaprawan C, Chenchittikul M, Udompanthurat S: Etiologies of acute undifferentiated febrile illness in Thailand. $J$ Med Assoc Thai 2004, 87(5):464-72.

10. Watt $G$, Jongsakul $K$ : Acute undifferentiated fever caused by infection with Japanese encephalitis virus. Am J Trop Med Hyg 2003, 68(6):704-6.

11. Gur H, Aviram R, Or J, Sidi Y: Unexplained fever in the ED: analysis of 139 patients. Am J Emerg Med 2003, 21(3):230-5.

12. Joshi R, Colford JM Jr, Reingold AL, Kalantri S: Nonmalarial acute undifferentiated fever in a rural hospital in central India: diagnostic uncertainty and overtreatment with antimalarial agents. Am J Trop Med Hyg 2008, 78(3):393-9.

13. Phuong HL, de Vries PJ, Nagelkerke N, Giao PT, Hung le Q, Binh TQ, Nga TT, Nam NV, Kager PA: Acute undifferentiated fever in Binh Thuan province, Vietnam: imprecise clinical diagnosis and irrational pharmaco-therapy. Trop Med Int Health 2006, 11(6):869-79.

14. Jones $A E$, Troyer $J L$, Kline JA: Cost-effectiveness of an emergency department-based early sepsis resuscitation protocol. Crit Care Med 2011, 39(6):1306-12.

15. Kikuchi T, Toba S, Sekiguchi Y, Iwashita T, Imamura H, Kitamura M, Nitta K, Mochizuki K, Okamoto K: Protocol-based noninvasive positive pressure ventilation for acute respiratory failure. J Anesth 2011, 25(1):42-9.

doi:10.1186/1865-1380-4-57

Cite this article as: Thangarasu et al:: A protocol for the emergency department management of acute undifferentiated febrile illness in India. International Journal of Emergency Medicine 2011 4:57.

\section{Submit your manuscript to a SpringerOpen ${ }^{\mathcal{O}}$ journal and benefit from:}

- Convenient online submission

- Rigorous peer review

- Immediate publication on acceptance

- Open access: articles freely available online

- High visibility within the field

- Retaining the copyright to your article

Submit your next manuscript at $\gg$ springeropen.com 